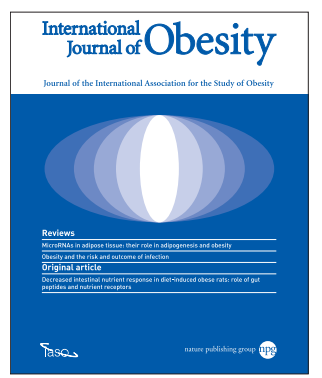

\section{Attenuation of diet-induced obesity and induction of white fat browning with a chemical inhibitor of histone deacetylases}

\author{
A Ferrari, E Fiorino, R Longo, S Barilla, N Mitro, G \\ Cermenati, M Giudici, D Caruso, A Mai, U Guerrini, E De \\ Fabiani, M Crestani
}

Cite this article as: A Ferrari, E Fiorino, R Longo, S Barilla, N Mitro, G Cermenati, M Giudici, D Caruso, A Mai, U Guerrini, E De Fabiani, M Crestani, Attenuation of diet-induced obesity and induction of white fat browning with a chemical inhibitor of histone deacetylases, International Journal of Obesity accepted article preview 31 October 2016; doi: 10.1038/ijo.2016.191.

This is a PDF file of an unedited peer-reviewed manuscript that has been accepted for publication. NPG are providing this early version of the manuscript as a service to our customers. The manuscript will undergo copyediting, typesetting and a proof review before it is published in its final form. Please note that during the production process errors may be discovered which could affect the content, and all legal disclaimers apply.

Received 24 February 2016; revised 16 September 2016; accepted 23 September 2016; Accepted article preview online 31 October 2016 


\section{ACCEPTED ARTICLE PREVIEW}

Attenuation of diet-induced obesity and induction of white fat browning with a chemical inhibitor of histone deacetylases

Running title: Class I HDACs regulates adipose tissue metabolism

Alessandra Ferrari $^{1}$, Erika Fiorino ${ }^{1}$, Raffaella Longo ${ }^{1}$, Serena Barilla ${ }^{1 \#}$, Nico Mitro ${ }^{1}$,

Gaia Cermenati ${ }^{1}$, Marco Giudici ${ }^{1}$, Donatella Caruso $^{1}$, Antonello Mai ${ }^{2}$, Uliano Guerrini $^{1}$, Emma De Fabiani ${ }^{1}$, Maurizio Crestani ${ }^{1 *}$.

${ }^{1}$ DiSFeB, Dipartimento di Scienze Farmacologiche e Biomolecolari, Università degli Studi di Milano, Milano, 20133, Italy;

${ }^{2}$ Dipartimento di Chimica e Tecnologie del Farmaco, Istituto Pasteur-Fondazione Cenci Bolognetti, Sapienza Università di Roma, Roma, Italy;

\#Current address: Karolinska Institutet, Institutionen för biovetenskaper och näringslära, Novum, hiss D, plan 7 Hälsovägen 7, S-14157 Huddinge

*Correspondence: Maurizio Crestani

Dipartimento di Scienze Farmacologiche e Biomolecolari, Università degli Studi di Milano

Via Balzaretti 9, 20133, Milano

Phone: +390250318393

Fax: +390250318391

maurizio.crestani@unimi.it

The authors declare no conflict of interest. 


\section{ABSTRACT}

Background/Objectives: In the last decade a strict link between epigenetics and metabolism has been demonstrated. Histone deacetylases (HDACs) have emerged as key epigenetic regulators involved in metabolic homeostasis in normal and pathologic conditions. Here we investigated the effect of the class I HDAC inhibitor MS-275 in a model of obesity induced by a high fat diet (HFD).

Methods: C57BL6/J male mice were fed HFD for 17 weeks and then randomized in two groups treated intraperitoneally with vehicle (DMSO) or with the class I selective HDAC inhibitor MS-275 every other day for 22 days. Glucose tolerance test and measurement of body temperature during cold exposure were performed. Adipose tissues and liver were phenotypically characterized through histological analysis. Gene and protein expression analysis of brown and white adipose tissues were performed.

Results: MS-275 treated mice showed 10\% reduction of body weight, lower adipocyte size, and improved glucose tolerance. Inhibition of class I HDAC determined reduction of adipocyte size and of fat mass, paralleled by higher expression of adipose functionality markers and by increased rate of lipolysis and fatty acid $\beta$-oxidation. MS-275 also promoted thermogenic capacity, related to "browning" of visceral and subcutaneous white adipose tissue, showing increased expression of uncoupling protein 1. In brown adipose tissue we observed limited effects on gene expression and only reduction of brown adipocyte size.

Conclusions: This study provides evidence that class I HDAC inhibition stimulated functionality and oxidative potential of adipose tissue, improving glucose tolerance and ameliorating the metabolic profile in diet-induced obese mice. 


\section{ACCEPTED ARTICLE PREVIEW}

\section{INTRODUCTION}

The prevalence of obesity increased at a worrisome rate worldwide and is predicted to continuously grow in the next two decades. The fast increase in the number of obese people is related to a sedentary lifestyle, accompanied by excessive energy intake not adequately counteracted by energy expenditure (1). Obesity is characterized by excessive accumulation of body fat and it is often associated with insulin resistance and the onset of type 2 diabetes (2). It is nowadays established that genetic susceptibility plays a fundamental role in the development of this disorder (3), however a role of epigenetics in the pathophysiology of metabolic disorders has recently emerged (4). Epigenetic modifications include DNA methylation and histone post-translational modifications, such as acetylation, methylation, ADP-ribosylation and phosphorylation (5). In the context of metabolic diseases a number of evidences highlighted the importance of histone acetylation, focusing the attention on the role of histone deacetylases (HDACs) (6-8). These enzymes, by deacetylating histones compact chromatin making it less accessible to transcriptional activators (9). Gao and colleagues demonstrated that sodium butyrate, a short chain fatty acid that inhibits class I and class II HDAC activity, improves diet induced obesity and diabetes (10). Class II HDACs affect both histone and non-histone proteins and butyrate inhibits both main classes of HDACs thus affecting energy metabolism also via HDACindependent mechanisms $(11,12)$; furthermore it has been shown that butyrate acts through free fatty acid receptors (FFAR2 and FFAR3) on the release of the intestinal factor glucagon-like peptide-1 (Glp1) $(13,14)$. Therefore, further investigations were undertaken to fully understand the role of HDACs in the regulation of energy metabolism and which class of HDACs is mainly involved in the modulation of metabolic processes. Our previous work demonstrated that in a genetic model of 


\section{ACCEPTED ARTICLE PREVIEW}

obesity and insulin resistance such as the $d b / d b$ mouse, the class I selective HDAC inhibitor MS-275 enhanced oxidative metabolism in skeletal muscle and adipose tissue and promoted energy expenditure, leading to reduction of body weight and improvement of insulin sensitivity (15). Interestingly, MS-275 was able to induce a metabolic switch of white adipose tissue (WAT) toward a more oxidative phenotype resembling the features of brown adipose tissue (BAT), a phenomenon referred to as "browning". Upon physiological stimuli such as chronic cold exposure (16), hormonal stimuli (e.g. irisin) (17), pharmacological treatment (thiazolidinediones) (18) or $\beta$-adrenergic stimulation (19), WAT switches the genetic program towards a brown-fat-like phenotype characterized by induced expression of uncoupling protein 1 (Ucp1), cell death-inducing DFFA-like effector a (Cidea), and diodinase 2 (Dio2). These inducible or recruitable brown adipocytes, also known as "brite" (brown in white) or beige adipocytes, compared to classical brown adipocytes show lower number of mitochondria and thermogenesis capacity at basal state; however, upon appropriate external cues, they become multilocular and increase number of mitochondria, resembling BAT phenotype (20). This morphological change, together with inducible expression of UCP1 and other genes related to mitochondrial biogenesis, results in a cell type that participates to adaptive thermogenesis and energy expenditure similar to classical BAT. It has been shown that browning of WAT is an efficient strategy to counteract obesity and diabetes in rodent models (21, 22).

Genetic obesity and diet-induced obesity differ in term of lipid metabolism and adipose tissue phenotype (23). Epidemiological studies showed that dietary habits is an important determinant of body weight in humans and that a diet enriched in fat increases the incidence of obesity (24). Moreover leptin resistance in humans is rarely 


\section{ACCEPTED ARTICLE PREVIEW}

related to a mutation in leptin gene; thus the use of murine models of obesity with dysfunctional leptin signaling ( $o b / o b$ or $d b / d b$ mice) could limit the translational relevance of the studies since it is not completely representative of human pathologic condition (25). Therefore, a diet-induced model of obesity may provide a framework that mimics more closely overweight humans and may be useful to study the pharmacology of obesity.

In order to evaluate whether biochemical inhibition of class I HDACs could be a promising strategy in the treatment of diet induced obesity, here we evaluated the effect of MS-275 in mice fed high fat diet. We found that this class I HDAC inhibitor reduced body weight and improved glucose tolerance. Interestingly, treatment with MS-275 increased thermogenic capacity and induced browning of both visceral and subcutaneous WAT. Our work demonstrated the crucial importance of class I HDACs in the pathophysiology of obesity, unraveling the role of these enzymes in the determination of metabolic signature of WAT.

\section{MATERIALS AND METHODS}

\section{$\underline{\text { Animal studies }}$}

For high fat diet feeding experiment, C57BL/6J male mice were purchased from The Jackson Laboratory. Six-week-old male mice were fed high fat diet (HFD, 60\% calories from fat, Research Diet, D12492) for 17 weeks, monitoring body weight and fasting glycaemia. At week 18, mice were randomized and treated for 22 days every other day with intraperitoneal injection of vehicle (DMSO) or MS-275 (10mg/kg). At the end of treatments, brown and white adipose tissues (inguinal subcutaneous and mesenteric visceral fat pads), liver and blood samples were collected from individual 


\section{ACCEPTED ARTICLE PREVIEW}

animals. For blood analysis, HFD fed mice were fasted for 16 hours and blood was collected from the tail vein. Glucose levels were determined using One touch Ultra ${ }^{\circledR}$ glucometer. Triglyceride and cholesterol levels were determined by Plasma Triglyceride Kit (Sentinel) and by Plasma Cholesterol Kit (Sentinel). Non-esterified fatty acids (NEFA) levels were determined by NEFA Kit (Wako Diagnostics). Insulin levels were determined using AlphaLISA ${ }^{\circledR}$ Human Insulin Research Immunoassay Kit (Perkin Elmer). Adiponectin and Leptin levels were determined using ELISA assay Kits (Genway). For glucose and insulin tolerance test, mice were fasted for 16 hours, and glucose levels from tail vein blood were determined before and 30, 60, 90, and $120 \mathrm{~min}$ after i.p. injection of glucose $(2 \mathrm{~g} / \mathrm{kg})$. For cold challenge test, basal rectal temperature was measured at $24^{\circ} \mathrm{C}$. Subsequently, mice were housed for 140 minutes at $4{ }^{\circ} \mathrm{C}$ and rectal temperature was measured every 20 minutes.

All experiments were conducted in accordance with Italian regulations (decree no. 116, 27 January 1992).

\section{$\underline{\text { Histological analysis of adipose tissues }}$}

Brown adipose tissue, subcutaneous white adipose tissue, and liver were fixed with Carnoy solution (6 parts 100\% ethanol, 3 parts chloroform, 1 part glacial acetic acid) for 24 hours at $4^{\circ} \mathrm{C}$. Tissues were then transferred to $100 \%$ ethanol. Tissues were embedded in paraffin and $8 \mu \mathrm{m}$ sections were stained with hematoxylin and eosin. Images were taken at 20X magnification. Cell size of adipocytes from white adipose tissue sections were quantitated using Photoshop CS6 (Adobe Systems Inc., San Jose, California).

\section{$\underline{\text { Gene expression }}$}




\section{ACCEPTED ARTICLE PREVIEW}

RNA from adipose tissues was isolated using Qiazol Reagent ${ }^{\circledR}$ (Qiagen), purified with commercial kit (RNeasy Lipid Tissue Mini kit, Qiagen), and quantitated with Nanodrop (Thermo Scientific, Wilmington, DE). Specific mRNA was amplified and quantitated by real time qPCR, using iScriptTM One Step RT PCR for Probes (BioRad Laboratories), following the manufacturer's instructions. Data were normalized to $36 \mathrm{~B} 4 \mathrm{mRNA}$ and quantitated setting up a standard curve.

Each sample was run in triplicate using 36B4 as reference gene. Primers and probes were obtained from Eurofins MWG Operon and are available upon request.

\section{$\underline{\text { Protein analysis }}$}

Adipose tissues were lysed with 2X SDS sample buffer (20\% glycerol, 4\% SDS, 100 $\mathrm{mM}$ Tris- $\mathrm{HCl} \mathrm{pH} 6.8,100 \mathrm{mM}$ dithiothreitol, $0.002 \%$ bromophenol blue), $1 \mathrm{mM}$ protease inhibitors (Sigma), and homogenized with TissueLyser (Qiagen). Tissue lysates were separated by SDS-PAGE, transferred onto nitrocellulose membranes, and sample loading and transfer were evaluated by Ponceau staining. Membranes were then blocked in 5\% non-fat dry milk in $1 \mathrm{X}$ TBS. The following primary antibodies were used: PPAR $\gamma$ (1:500, sc-7273, Santa Cruz), UCP1 (1:1000, ab10983, Abcam). HRP-conjugated goat anti-mouse (Sigma-Aldrich) and goat anti-rabbit (Cell Signaling) secondary antibodies were used for detection with chemiluminescence (ECL, Pierce).

\section{$\underline{\text { Immunohistochemistry }}$}

Sections of WAT and BAT $(8 \mu \mathrm{m})$ were deparaffinized and antigen retrieval was performed with $0.05 \mathrm{M} \mathrm{NH} \mathrm{Nl}_{4}$ for $30 \mathrm{~min}$ at room temperature. Endogenous peroxidase activity was blocked with $1 \% \mathrm{H}_{2} \mathrm{O}_{2}$ for 20 min. Blocking was performed 


\section{ACCEPTED ARTICLE PREVIEW}

in $1 \%$ BSA-0.1\% Triton X-100 for $1 \mathrm{~h}$. Anti-UCP1 (Abcam) was applied (1:300) overnight at $4{ }^{\circ} \mathrm{C}$, followed by incubation with biotinylated secondary antibodies (1:3000). Histochemical reactions were performed using diaminobenzidine. Quantification of histochemical analysis was performed counting the number of pixels in gray scale intervals, using the software ImageJ. Color channels were split and the analysis was performed on green channel. The software generates the list of gray scale intervals (0-255) and the number of pixels in each interval.

\section{Magnetic resonance imaging}

At day 18 of treatment, mice were anesthetized and analyzed in a 4.7 Tesla Avance II magnetic resonance imaging (MRI) scanner (Bruker Corporation). After a gradientecho scout, 16 axial 1-mm-thick T1-weighted slices were placed in the abdominal region spanning from kidneys to bladder inclusive. The field of view was 30 X 30 mm2 with a matrix of $128 \times 128$ pixels. Four averages of a spin echo sequence with echo time to $10 \mathrm{~ms}$ and repetition time $400 \mathrm{~ms}$ were acquired in 3'25". The slice immediately frontal with respect to the ilium bone was chosen for visceral fat estimation and was computed as follows: (fat area)/(slice area). Areas were measured with Photoshop (Adobe Systems).

\section{Mass spectrometry analysis of hepatic fatty acids}

Livers from HFD fed obese mice treated with vehicle or with MS-275 were homogenized in methanol with tissue lyser (Qiagen). For quantitative analysis of fatty acids, aliquots of methanolic extracts after addition of internal standards (heneicosanoic acid, C21:0, and ${ }^{13} \mathrm{C}$-labeled linoleic acid, Sigma-Aldrich) were subjected to acid hydrolysis and processed as previously described (26). Fatty acid 


\section{ACCEPTED ARTICLE PREVIEW}

quantification was performed on an API-4000 triple quadrupole mass spectrometer (AB SCIEX) coupled with a HPLC system (Agilent) and CTC PAL HTS autosampler (PAL System).

\section{$\underline{\text { Human adipose stromal cells (hASC) }}$}

Cells were plated at a density of $10^{5}$ cell $/ \mathrm{ml}$ and differentiated for 4 days in presence of $50 \mu \mathrm{M}$ insulin, $100 \mathrm{nM}$ dexamethasone, $0.25 \mathrm{mM}$ isobutylmethylxantine (Sigma Aldrich), 100nM rosiglitazone (Cayman Chemical). Then medium was refreshed every other day from day 4 to day 10 of differentiation with of $50 \mu \mathrm{M}$ insulin, $100 \mathrm{nM}$ dexamethasone (Sigma Aldrich), 100nM rosiglitazone (Cayman Chemical). At the end of differentiation cells were treated for 48 hours with DMSO or with $1 \mu \mathrm{M}$ MS275.

$\underline{\text { Statistical analyses }}$

Statistical analyses were performed using the unpaired two-tailed Student $t$ test with GraphPad PRISM (San Diego, CA).

\section{RESULTS}

Inhibition of class I HDACs improves obese phenotype and reduces adiposity

To investigate whether inhibition of class I HDACs could counteract obesity and insulin resistance in a mouse model of diet-induced obesity (DIO mice), we fed 6week-old male C57BL/6J mice with high fed diet (HFD, 60\% kcal from fat). After 17 weeks on HFD mice were randomized in two groups, without statistically significant difference in weight and fasting glycaemia. Mice were treated for 22 days, every other 


\section{ACCEPTED ARTICLE PREVIEW}

day, with vehicle DMSO or with the class I selective HDAC inhibitor MS-275

$10 \mathrm{mg} / \mathrm{kg}$ (Figure 1A), as previously reported (15). Starting from day 16 of treatment, MS-275 treated mice showed a lower body weight (Figure 1B), yielding 10\% reduction at day 22, with no statistically significant change in daily food intake (Supplementary Figure 1A). Body weight of each mouse before and after the treatments was reported in Table 1. MS-275 decreased the percentage of total WAT (Figure 1C, Supplementary Figure 1E) and in particular MRI analysis showed statistical significant reduction of subcutaneous WAT (Supplementary Figure 1B) and a mild but not statistically significant decrease of visceral WAT (Supplementary Figure 1C). No difference of brown adipose tissue (BAT) mass was detected in mice treated with MS-275 (Supplementary Figure 1D). Glucose tolerance test showed that treatment with MS-275 improved glucose clearance in diet induced obese mice (Figure 1D), while insulin tolerance test showed no differences between the two groups (Supplementary Figure 1J). MS-275 was also able to improve leptin resistance as assessed by reduced levels of circulating leptin and by the increased expression of hypothalamic leptin receptor (Supplementary Figure 1F, 1G), while it had no effect on circulating levels of insulin and adiponectin (Supplementary Figure 1H, 1I). Hematoxylin and eosin staining of subcutaneous (inguinal) and visceral (mesenteric) WAT revealed that adipocytes were smaller in MS-275-treated mice (Figure 1E).

MS-275 increased expression of markers of lipid catabolism and adipose functionality To explain the phenotypic effects observed in MS-275 treated mice we analyzed the gene expression profile in their adipose tissue depots. Interestingly, we found that MS-275 increased expression of the master regulator of adipogenesis Pparg and of other typical markers of adipose tissue functionality (Fabp4, Adipoq, Glut4, Plin) 


\section{ACCEPTED ARTICLE PREVIEW}

(Figure 2A, 2B). The augmented expression of most of these genes (except Glut4) was statistically significant in the visceral WAT whereas, in spite of the general trend, only Glut4 and Plin resulted significantly enhanced in subcutaneous WAT. Of note, western blot analysis of subcutaneous WAT revealed strong increase in PPAR $\gamma$

protein expression (Figure 2C, Supplementary Figure 1K), suggesting a posttranscriptional modulation of this protein in response to class I HDAC inhibition. We also found that class I HDAC inhibitor increased the expression of genes involved in lipolysis (Atgl) and fatty acid $\beta$-oxidation (Cptlb, Acadl) in both subcutaneous (Figure 2D) and visceral (Figure 2E) WAT. We hypothesized that the reduced lipid content in white adipocytes is the result of a higher catabolism, and that it is not related to an inefficient storage of lipids in WAT. In line with this observation, we did not detect an increase in circulating levels of triglycerides (Supplementary Figure 2A) and cholesterol (Supplementary Figure 2B). Measurement of NEFA revealed mild, though not statistically significant, increase in MS-275 group (Figure 2F). Moreover, we did not observe differences in the level of fatty acids in the liver of MS-275 treated mice, as detected by hematoxylin and eosin staining (Supplementary Figure 2C) and by mass spectrometry analysis (Supplementary Figure 2D), indicating that there is no ectopic lipid deposition in the liver in response to the HDAC inhibitor. To rule out the contribution of skeletal muscle to the metabolic profile in MS-275 treated mice, we analyzed expression of genes regulating metabolism in gastrocnemius (Supplementary Figure 2E) and found no changes in the expression of Ppargcla, Pppargclb and of cytochrome $\mathrm{C}(\mathrm{Cyt} C)$. On the other hand, we detected increased expression of mitochondrial biogenesis genes Tfam and Tfblm (Supplementary Figure 2E). Interestingly gene encoding for the ETC protein COX7A1 was significantly reduced, suggesting no increase in oxidative potential upon MS-275 treatment in 


\section{ACCEPTED ARTICLE PREVIEW}

skeletal muscle. Moreover, we found reduced expression of Glut4, indicating that amelioration of systemic glucose tolerance is not related to improved glucose uptake in skeletal muscle.

\section{$\underline{\text { Inhibition of class I HDACs increases thermogenic capacity }}$}

Previous results in $d b / d b$ mice demonstrated that class I HDAC inhibition increased heat production (15). Thus we investigated whether treatment with MS-275 could affect thermogenic capacity in diet induced obese mice. After acute exposure to $4^{\circ} \mathrm{C}$ MS-275 treated mice were able to better cope the cold challenge (Figure 3A). The organ most involved in the regulation of body temperature via non shivering thermogenesis is BAT (27). Magnetic resonance imaging of interscapular area revealed that BAT mass was not affected by MS-275 treatment (Supplementary Figure 1D). Hematoxylin and eosin staining in BAT from control and MS-275 treated mice showed that the HDAC inhibitor altered tissue morphology (Figure 3B, top panels), apparently reducing lipid accumulation, typically increased in BAT from obese mice. However, immunohistochemical analysis of UCP1 showed no differences in vehicle and MS-275 treated mice (Figure 3B, bottom panels). Accordingly, gene expression analysis did not show changes in the mRNA and protein expression of the key regulator of thermogenesis UCP1 (Figure 3C, 3D) and of the other genes typically expressed in brown fat (Adrb3, Cidea, Prdm16) (Figure 3C). Interestingly we detected an increased expression of typical BAT markers Dio2 and Elovl3.

Inhibition of class I HDACs promotes lipid oxidation and browning in white fat

Since we found increased heat production during cold exposure but we did not observe higher expression of UCP1 in BAT of mice treated with the class I HDAC 


\section{ACCEPTED ARTICLE PREVIEW}

inhibitor, we wanted to explore whether MS-275 was able to induce a brown-like phenotype in WAT also in a model of diet induced obesity. Interestingly, mice treated with HDAC inhibitor showed clear signs of browning of both subcutaneous (Figure 4A) and visceral WAT (Figure 4B), since Ucpl and other genes typically expressed in BAT (Adrb3, Cidea, Dio2, Ppara) were upregulated. Immunohistochemical analysis of UCP1 confirmed its higher expression in subcutaneous (Figure 4C) and visceral fat (Figure 4D) of MS-275 treated mice. To deeply investigate the phenotypical rearrangement occurring upon class I HDAC inhibition in white adipose tissues we performed further gene expression analysis and we found upregulation of markers of fatty acid synthesis: fatty acid synthase (Fasn) was increased in both subcutaneous WAT and visceral WAT, while acetyl CoA carboxylase (Acaca) was significantly upregulated in visceral WAT (Supplementary Figure 3A, 3B). Moreover the gene expression analysis of inflammation markers revealed no differences in the mRNA of M1 markers $i N O S$ and $I L 1 \beta$ in adipose tissues (Supplementary Figure 3C, 3D), and a significant increase in subcutaneous WAT of markers of alternatively activated macrophages (M2) markers $M R C 1$ and $C d 163$. We also measured levels of apoptosis markers (Caspases) but found no changes in MS-275 treated mice (Figure Supplementary Figure 3E, 3F), thus ruling out the contribution of apoptosis in white fat in body weight reduction.

To extend our observations in murine models to the human settings, we treated differentiated human adipose stromal cells (hASC) with vehicle (DMSO) or with MS275 for 48 hours: we detected increased rate of differentiation in presence of class I HDAC inhibitor as assessed by Oil Red O staining (Supplementary Figure 3G, 3H). Although this preliminary observation suggests a possible role of class I HDACs in human adipose tissue physiology, further investigations will be required to assess 


\section{ACCEPTED ARTICLE PREVIEW}

whether modulation of class I HDACs activity may reprogram adipocyte metabolism in humans.

\section{DISCUSSION}

Human obesity is often related to a diet enriched in fat and carbohydrates in Western countries (24). To provide new knowledge about metabolic disorders consequent to an unhealthy intake of calories from fat the most useful murine model is a dietinduced model of obesity. It has in fact been shown that the use of genetic models of obesity with dysfunctional leptin signaling (ob/ob or $d b / d b$ mice) could not be completely representative of human pathologic condition, considering that genetic mutations of leptin gene are rare in obese patients. Previous investigations showed that treatment of diet induced obese mice with HDAC inhibitor sodium butyrate improved insulin sensitivity by promoting energy metabolism in skeletal muscle and brown adipose tissue (10). However, the short chain fatty acid butyrate may display effects also through non-HDAC targets (e.g. FFAR2 and FFAR3) $(13,14)$. Therefore, in this study we decided to adopt a murine model of diet-induced obesity (DIO) to investigate the role of HDACs in this pathological condition, using the synthetic class I HDAC selective inhibitor MS-275. Here we reported that inhibition of class I HDACs by MS-275 reduces body weight and enhances glucose tolerance. The fact that treatment with MS-275 improved GTT but did not affect ITT could be ascribed to higher expression of Glut4 in adipose tissues resulting in amelioration of the capacity to handle glucose loads, rather than to increased insulin signaling and sensitivity. Moreover, class I HDAC inhibitor increased thermogenic capacity. This result prompted us to investigate further the effects of class I HDAC inhibitor in BAT. Although BAT mass did not change, we found that BAT morphology was improved 


\section{ACCEPTED ARTICLE PREVIEW}

in MS-275 treated mice, which showed higher number of small multilocular brown adipocytes, expressing BAT markers such as Dio2 and Elovl3. Interestingly no significant changes were found in the expression of Ucpl and Adrb3 thus arguing against increased heat production by BAT following MS-275 treatment. The two proteins encoded by these genes, uncoupling protein 1 and $\beta_{3}$ adrenergic receptor, are in fact considered the key activators of the thermogenic program. However, we cannot completely exclude the contribution of BAT to increased thermogenesis in MS-275 treated mice since an important contribution of DIO2 to thermogenesis has been reported (28): Dio2-/- mice during cold exposure become hypothermic, despite normal expression of UCP1, demonstrating that type 2 iodothyronine deiodinase and uncoupling protein 1 regulate thermogenesis in a concerted fashion. Thus, we do not rule out that long-term exposure of MS-275 treated mice to cold could result in an increase of BAT uncoupling and functionality. However, an explanation for the increased thermogenic activation in response to acute cold exposure in MS-275 treated mice came from the analysis of white fat. Our results indicate, in fact, that MS-275-elicited browning of WAT contributes in counteracting diet-induced obesity. As previously reported, browning of WAT occurring without changes of uncoupling protein 1 in BAT, is a successful strategy to counteract obesity (17), (29), (30). Interestingly, in both subcutaneous and visceral WAT from MS-275 treated mice we observed upregulation of Ucpl and other markers of BAT. Thus "browning" of white adipose tissue consequent to inhibition of class I HDACs in DIO mice occurred both in subcutaneous and visceral WAT. Accordingly, expression of WAT functionality markers and of genes involved in triglycerides and fatty acid catabolism were upregulated in both types of white fat, though to a greater extent in visceral WAT. To date, browning of WAT has been obtained by pharmacological treatment or by 


\section{ACCEPTED ARTICLE PREVIEW}

genetic manipulation $(18,20,31)$ in inguinal WAT or in epidydimal WAT. Our data, showing browning in visceral mesenteric WAT, suggest that class I HDAC selective inhibition induces deep reprogramming of adipose phenotype, resulting in more functional visceral adipocytes. Recently, it has been demonstrated that leptin mediates white adipose tissue browning through sympathetic nerve activity on adipose tissue through proopiomelanocortin (POMC) neurons (32). Interestingly, leptin levels were reduced in MS-275 treated mice, thus we hypothesize that these mice are more sensitive to leptin. Gene expression analysis in hypothalamus seems to corroborate this interpretation, as leptin receptor mRNA levels were higher in mice treated with the HDAC inhibitor MS-275. Thus, based on our results (higher expression of leptin receptor leading to increased leptin sensitivity in hypothalamus in mice treated with MS-275) and on the published literature, it is possible that leptin plays a role in MS275-mediated browning of white adipose tissue. Gene expression analysis indicates that white adipocytes of MS-275 treated mice could catabolize triglycerides at higher rate as indicated by increased expression of adipose tissue lipase (Atgl). Typically, high lipolytic rate correlates with increased level of plasma NEFA, however, in our experimental settings circulating NEFA are not statistically different in two groups of mice. In MS-275 treated mice this is probably the result of enhanced fatty acid $\beta$ oxidation within WAT depots, that use fatty acids as a metabolic fuel, as proved by increased expression of $\beta$-oxidation genes (i.e. Ppara, Acadl, Cptlb). Similarly to what happens to brown adipose tissue during cold exposure (33) and to white adipose tissue upon $\beta_{3}$-adrenergic stimulation (34), MS-275 also boosted de novo fatty acid synthesis in subWAT and viscWAT, further confirming the browning effect mediated by class I HDAC inhibitor. We infer that activation of lipogenic program in brownlike WAT might be an adaptive response to the increased requirement of fuel 


\section{ACCEPTED ARTICLE PREVIEW}

molecules to feed the accelerated electron transport chain (ETC) flux due to uncoupling of ETC from oxidative phosphorylation. The finding of improved functionality of mesenteric white adipose tissue is particularly relevant when considering the link between the intra-abdominal fat accumulation typical of obesity and other related severe complications, such as insulin resistance/type 2 diabetes and ultimately cardiovascular events. In spite of these considerations the MRI analysis revealed a significant reduction of adipose mass in subcutaneous WAT whereas in visceral WAT the trend did not reach statistical significance. This could depend on different features of viscWAT and subWAT: subcutaneous fat, which is less susceptible to inflammation (35), is characterized by larger adipocytes and higher plasticity as compared to visceral fat. MS-275 reduced the size of both subcutaneous and visceral adipocytes, however the effect was more pronounced in subWAT. This dramatic reduction of subcutaneous adipocytes hypertrophy can explain the reduced subWAT mass, as detected by MRI analysis. Conversely, gene expression analysis of functionality markers showed that MS-275 is more efficient in visceral fat: visceral adipocytes are smaller than subcutaneous adipocytes, yet visceral fat is more susceptible to inflammation because of the lower critical size adipocytes in this depot (35). Thus, even though the reduction in adipocyte size is not as evident as in subWAT, the decrease of adipocyte hypertrophy is sufficient to rescue adipocyte functionality, as assessed by gene expression analysis. On the contrary, subcutaneous adipocytes in obesity feature greater expandability that does not impair their functionality. For this reason, the effects of MS-275 on the expression of functionality markers are less pronounced in subWAT as compared to the effects observed in viscWAT. In both subcutaneous and visceral WAT, MS-275 has no effect on adipose tissue inflammation typically associated to obesity, as assessed by unaltered 


\section{ACCEPTED ARTICLE PREVIEW}

expression of pro-inflammatory macrophage (M1) markers. However, in subWAT inhibition of class I HDAC is able to induce the expression of alternatively activated macrophages (M2). The increased expression of these markers further corroborate the browning phenotype, since it has been reported that M2 macrophages produce cathecolamines that sustain adaptive thermogenesis activation (36), driving the development of cold-induced beige fat in inguinal WAT (37). Furthermore, the unchanged expression level of Caspases in subWAT and viscWAT highlights that inhibition of class I HDAC does not promote adipocytes apoptosis.

Assuming that HDAC inhibitors do not act on specific organs but exert ubiquitous effects, we cannot exclude that MS-275 affects metabolism and physiology of other organs. At this regard, we looked at skeletal muscle gene expression and found that in gastrocnemius treatment with MS-275 slightly increased the expression of mitochondrial biogenesis genes (Tfam, $T f b 1 m$ ) but it decreased expression of insulin dependent glucose transporter Glut4. This result might indicate that amelioration of systemic glucose tolerance is probably not related to improved glucose utilization in skeletal muscle, but to ameliorated glucose clearance by adipose tissue. It should be noted that selective Glut4 overexpression in adipose tissue prevents glucose intolerance in mice fed high fat diet (38); consistently, in our experimental model MS-275 increased Glut4 expression in subcutaneous WAT, which is likely to contribute to improved glucose tolerance.

Another organ that may contribute to glucose tolerance is liver. However, histological images and mass spectrometry analysis did not show significant difference in hepatic lipid levels between control and MS-275 treated mice. Therefore we tend to exclude that the liver may play a role in improving glucose clearance in our experimental model. 


\section{ACCEPTED ARTICLE PREVIEW}

It has been reported that hepatic deletion of $\operatorname{HDAC} 3(39,40)$ leads to lipid accumulation in the liver. However, although MS-275 targets both HDAC1 and HDAC3, we found no hepatic steatosis in the liver of DIO mice treated with this inhibitor. This difference may be ascribed to the effect of global versus local HDAC3 inhibition: in obese and diabetic mice, systemic HDAC3 inhibition (i.e., MS-275 treatment) increases oxidative metabolism and energy expenditure in other tissues such as adipose tissue, thus preventing hepatic lipid buildup.

The preliminary observations in human differentiated adipocytes highlight the role of class I HDACs in the regulation of adipocyte differentiation and functions in the human settings. Further analyses will shed light whether these effects result in a metabolic switch of human white adipocytes and whether HDAC inhibition could be evaluated as a possible strategy in the treatment of metabolic disorders.

In conclusion, our results demonstrate that inhibition of class I HDACs ameliorates obese and diabetic phenotype in a model of diet-induced obesity. This is a consequence of improved functionality of adipose tissue, preventing the excessive lipid accumulation that would impair adipocyte function and lead to insulin resistance. The role of class I histone deacetylases in adipose tissue patho-physiology could be further explored in the quest of new therapeutic avenues for obesity and T2D.

\section{ACKNOWLEDGEMENTS}

We would like to thank Dr. Gabriele Campana (Università degli Studi di Bologna) for providing us hASC and the other members of the laboratory for valuable discussion. We are grateful with Elda Desiderio Pinto for administrative support. This research 


\section{ACCEPTED ARTICLE PREVIEW}

was supported by grants from FP6 LSHM-CT2006-037498 and PRIN 2009K7R7NA to $\mathrm{MC}$.

\section{CONFLICT OF INTEREST}

The authors report no potential conflicts of interest relevant to this article.

Supplementary information is available at International Journal of Obesity's website. 


\section{REFERENCES}

1. Greener J, Douglas F, van Teijlingen E. More of the same? Conflicting perspectives of obesity causation and intervention amongst overweight people, health professionals and policy makers. Social Science \& Medicine. 2010;70(7):1042-9.

2. Nolan CJ, Damm P, Prentki M. Type 2 diabetes across generations: from pathophysiology to prevention and management. The Lancet. 2011;378(9786):16981.

3. Kopelman PG. Obesity as a medical problem. Nature. 2000;404(6778):635-

43.

4. Keating ST, El-Osta A. Epigenetic changes in diabetes. Clinical Genetics. 2013;84(1):1-10.

5. Vaillant I, Paszkowski J. Role of histone and DNA methylation in gene regulation. Current Opinion in Plant Biology. 2007;10(5):528-33.

6. Gray SG, Ekström TJ. The Human Histone Deacetylase Family. Experimental Cell Research. 2001;262(2):75-83.

7. Pham T, Lee J. Dietary Regulation of Histone Acetylases and Deacetylases for the Prevention of Metabolic Diseases. Nutrients. 2012;4(12):1868-86.

8. Ferrari A, Fiorino E, Giudici M, Gilardi F, Galmozzi A, Mitro N, et al. Linking epigenetics to lipid metabolism: Focus on histone deacetylases. Molecular Membrane Biology. 2012;0(0):1-10.

9. López-Rodas G, Brosch G, Georgieva EI, Sendra R, Franco L, Loidl P. Histone deacetylase: A key enzyme for the binding of regulatory proteins to chromatin. FEBS Letters. 1993;317(3):175-80.

10. Gao Z, Yin J, Zhang J, Ward RE, Martin RJ, Lefevre M, et al. Butyrate Improves Insulin Sensitivity and Increases Energy Expenditure in Mice. Diabetes. 2009;58(7):1509-17.

11. Donohoe DR, Garge N, Zhang X, Sun W, O'Connell TM, Bunger MK, et al. The Microbiome and Butyrate Regulate Energy Metabolism and Autophagy in the Mammalian Colon. Cell metabolism. 2011;13(5):517-26.

12. Haberland M, Montgomery RL, Olson EN. The many roles of histone deacetylases in development and physiology: implications for disease and therapy. Nat Rev Genet. 2009;10(1):32-42.

13. Nohr MK, Pedersen MH, Gille A, Egerod KL, Engelstoft MS, Husted AS, et al. GPR41/FFAR3 and GPR43/FFAR2 as cosensors for short-chain fatty acids in enteroendocrine cells vs FFAR3 in enteric neurons and FFAR2 in enteric leukocytes. Endocrinology. 2013;154(10):3552-64.

14. Tolhurst G, Heffron H, Lam YS, Parker HE, Habib AM, Diakogiannaki E, et al. Short-Chain Fatty Acids Stimulate Glucagon-Like Peptide-1 Secretion via the GProtein-Coupled Receptor FFAR2. Diabetes. 2012;61(2):364-71.

15. Galmozzi A, Mitro N, Ferrari A, Gers E, Gilardi F, Godio C, et al. Inhibition of Class I Histone Deacetylases Unveils a Mitochondrial Signature and Enhances Oxidative Metabolism in Skeletal Muscle and Adipose Tissue. Diabetes. 2012.

16. Vitali A, Murano I, Zingaretti MC, Frontini A, Ricquier D, Cinti S. The adipose organ of obesity-prone C57BL/6J mice is composed of mixed white and brown adipocytes. J Lipid Res. 2012;53(4):619-29.

17. Bostrom P, Wu J, Jedrychowski MP, Korde A, Ye L, Lo JC, et al. A PGC1alpha-dependent myokine that drives brown-fat-like development of white fat and thermogenesis. Nature. 2012;481(7382):463-8. 
18. Petrovic N, Walden TB, Shabalina IG, Timmons JA, Cannon B, Nedergaard J. Chronic Peroxisome Proliferator-activated Receptor $\gamma$ (PPAR $\gamma$ ) Activation of Epididymally Derived White Adipocyte Cultures Reveals a Population of Thermogenically Competent, UCP1-containing Adipocytes Molecularly Distinct from Classic Brown Adipocytes. Journal of Biological Chemistry. 2010;285(10):7153-64.

19. Cousin B, Cinti S, Morroni M, Raimbault S, Ricquier D, Penicaud L, et al. Occurrence of brown adipocytes in rat white adipose tissue: molecular and morphological characterization. Journal of Cell Science. 1992;103(4):931-42.

20. Harms M, Seale P. Brown and beige fat: development, function and therapeutic potential. Nat Med. 2013;19(10):1252-63.

21. Seale P, Conroe HM, Estall J, Kajimura S, Frontini A, Ishibashi J, et al. Prdm16 determines the thermogenic program of subcutaneous white adipose tissue in mice. J Clin Invest. 2011;121(1):96-105.

22. Bartelt A, Heeren J. Adipose tissue browning and metabolic health. Nat Rev Endocrinol. 2014;10(1):24-36.

23. Libinaki R, Heffernan M, Jiang WJ, Ogru E, Ignjatovic V, Gianello R, et al. Effects of genetic and diet-induced obesity on lipid metabolism. IUBMB Life. 1999;48(1):109-13.

24. Riccardi G, Giacco R, Rivellese AA. Dietary fat, insulin sensitivity and the metabolic syndrome. Clinical Nutrition. 2004;23(4):447-56.

25. Fellmann L, Nascimento AR, Tibiriça E, Bousquet P. Murine models for pharmacological studies of the metabolic syndrome. Pharmacology \& Therapeutics. 2013;137(3):331-40.

26. Cermenati G, Abbiati F, Cermenati S, Brioschi E, Volonterio A, Cavaletti G, et al. Diabetes-induced myelin abnormalities are associated with an altered lipid pattern: protective effects of LXR activation. Journal of Lipid Research.

2012;53(2):300-10.

27. Cypess AM, Lehman S, Williams G, Tal I, Rodman D, Goldfine AB, et al. Identification and Importance of Brown Adipose Tissue in Adult Humans. N Engl J Med. 2009;360(15):1509-17.

28. de Jesus LA, Carvalho SD, Ribeiro MO, Schneider M, Kim S-W, Harney JW, et al. The type 2 iodothyronine deiodinase is essential for adaptive thermogenesis in brown adipose tissue. The Journal of Clinical Investigation.108(9):1379-85.

29. Bi P, Shan T, Liu W, Yue F, Yang X, Liang X-R, et al. Inhibition of Notch signaling promotes browning of white adipose tissue and ameliorates obesity. Nat Med. 2014;20(8):911-8.

30. Shahid M, Javed AA, Chandra D, Ramsey HE, Shah D, Khan MF, et al. IEX-1 deficiency induces browning of white adipose tissue and resists diet-induced obesity. Scientific Reports. 2016;6:24135.

31. Tiraby C, Tavernier G, Lefort C, Larrouy D, Bouillaud F, Ricquier D, et al. Acquirement of Brown Fat Cell Features by Human White Adipocytes. J Biol Chem. 2003;278(35):33370-6.

32. Dodd GT, Decherf S, Loh K, Simonds SE, Wiede F, Balland E, et al. Leptin and insulin act on POMC neurons to promote the browning of white fat. Cell. 2015;160(1-2):88-104.

33. Trayhurn P. Fatty acid synthesis in mouse brown adipose tissue the influence of environmental temperature on the proportion of whole-body fatty acid synthesis in brown adipose tissue and the liver. Biochimica et Biophysica Acta (BBA) - Lipids and Lipid Metabolism. 1981;664(3):549-60. 


\section{ACCEPTED ARTICLE PREVIEW}

34. Mottillo EP, Balasubramanian P, Lee Y-H, Weng C, Kershaw EE, Granneman JG. Coupling of lipolysis and de novo lipogenesis in brown, beige, and white adipose tissues during chronic $\beta 3$-adrenergic receptor activation. Journal of Lipid Research. 2014;55(11):2276-86.

35. Murano I, Barbatelli G, Parisani V, Latini C, Muzzonigro G, Castellucci M, et al. Dead adipocytes, detected as crown-like structures, are prevalent in visceral fat depots of genetically obese mice. Journal of Lipid Research. 2008;49(7):1562-8.

36. Nguyen KD, Qiu Y, Cui X, Goh YP, Mwangi J, David T, et al. Alternatively activated macrophages produce catecholamines to sustain adaptive thermogenesis. Nature. 2011;480(7375):104-8.

37. Qiu Y, Nguyen KD, Odegaard JI, Cui X, Tian X, Locksley RM, et al.

Eosinophils and type 2 cytokine signaling in macrophages orchestrate development of functional beige fat. Cell. 2014;157(6):1292-308.

38. Herman MA, Peroni OD, Villoria J, Schon MR, Abumrad NA, Bluher M, et al. A novel ChREBP isoform in adipose tissue regulates systemic glucose metabolism. Nature. 2012;484(7394):333-8.

39. Knutson SK, Chyla BJ, Amann JM, Bhaskara S, Huppert SS, Hiebert SW. Liver-specific deletion of histone deacetylase 3 disrupts metabolic transcriptional networks. EMBO J. 2008;27(7):1017-28.

40. Sun Z, Miller RA, Patel RT, Chen J, Dhir R, Wang H, et al. Hepatic Hdac3 promotes gluconeogenesis by repressing lipid synthesis and sequestration. Nat Med. 2012;18(6):934-42.

\section{FIGURE LEGENDS}

Figure 1: Inhibition of class I HDACs ameliorates diet-induced obesity

a) Experimental paradigm in $\mathrm{C} 57 \mathrm{BL} / 6 \mathrm{~J}$ male mice

b) Body weight during pharmacological treatment in obese mice treated with vehicle

DMSO or with MS-275 (n=15 per group).

c) MRI analysis of abdominal (subcutaneous and visceral) fat of obese mice treated with vehicle DMSO or with MS-275 ( $\mathrm{n}=7$ per group).

d) Intra-peritoneal glucose tolerance test in obese mice treated with vehicle DMSO or with MS-275 (n=6 per group).

e) Hematoxylin and eosin staining and adipocyte size calculation of subcutaneous and visceral WAT obese mice treated with vehicle DMSO or with MS-275 ( $\mathrm{n}=5$ per group). Scale bar is $100 \mu \mathrm{m}$. 


\section{ACCEPTED ARTICLE PREVIEW}

Data are presented as mean \pm SEM. Statistical analysis: Student's t test, ${ }^{*} \mathrm{p}<0.05$, $* * * \mathrm{p}<0.001$.

Figure 2: MS-275 increases expression of markers of lipid catabolism and adipose functionality in WAT

a) Gene expression profile of markers of adipose functionality in subcutaneous WAT of obese mice treated with vehicle DMSO or with MS-275 ( $n=15$ per group)

b) Gene expression profile of markers of adipose functionality in visceral WAT of obese mice treated with vehicle DMSO or with MS-275 ( $\mathrm{n}=8-10$ per group)

c) Western blot analysis of PPAR $\gamma$ in subcutaneous WAT of obese mice treated with vehicle DMSO or with MS-275 (see also longer exposure in Supplementary Figure 1I).

d) Gene expression profile of markers of triglyceride catabolism and fatty acid $\beta$ oxidation in subcutaneous WAT of obese mice treated with vehicle DMSO or with MS-275 ( $\mathrm{n}=15$ per group)

e) Gene expression profile of markers of triglyceride catabolism and fatty acid $\beta$ oxidation in visceral WAT of obese mice treated with vehicle DMSO or with MS-275

f) Plasma NEFA levels in DIO mice treated with vehicle DMSO or with MS-275 ( $\mathrm{n}=15$ per group).

Data are presented as mean \pm SEM. Statistical analysis: Student's $t$ test, ${ }^{*} \mathrm{p}<0.05$, $* * \mathrm{p}<0.01, * * * \mathrm{p}<0.001$

Figure 3: Class I HDAC inhibition promotes thermogenesis

a) Body temperature of obese mice treated with vehicle DMSO or with MS-275 exposed to $4^{\circ} \mathrm{C}$ for 140 minutes ( $\mathrm{n}=7$ per group). 


\section{ACCEPTED ARTICLE PREVIEW}

b) Top panels: hematoxylin/eosin staining of BAT of obese mice treated with vehicle DMSO or with MS-275. Bottom panels: immunohistochemical analysis and of UCP1 in BAT of obese mice treated with vehicle DMSO or with MS-275. Scale bar is 100 $\mu \mathrm{m}$.

c) Gene expression analysis of BAT markers in brown fat of obese mice treated with vehicle DMSO or with MS-275 ( $\mathrm{n}=15$ per group).

d) Western blot analysis and quantification of UCP1 in BAT of obese mice treated with vehicle DMSO or with MS-275.

Data are presented as mean \pm SEM. Statistical analysis: Student's $t$ test, ${ }^{*} p<0.05$, $* * \mathrm{p}<0.01, * * * \mathrm{p}<0.001$

Figure 4: MS-275 induces browning of subcutaneous and visceral WAT

a) Gene expression analysis of BAT markers in subcutaneous WAT of obese mice treated with vehicle DMSO or with MS-275 ( $\mathrm{n}=15$ per group).

b) Gene expression analysis of BAT markers in visceral WAT of obese mice treated with vehicle DMSO or with MS-275 ( $n=8-10$ per group).

c) Immunohistochemical analysis and quantification of UCP1 in subcutaneous WAT of obese mice treated with vehicle DMSO or with MS-275 ( $n=3$ per group). Scale bar is $100 \mu \mathrm{m}$.

d) Immunohistochemical analysis and quantification of UCP1 in visceral WAT of obese mice treated with vehicle DMSO or with MS-275 ( $n=3$ per group). Scale bar is $100 \mu \mathrm{m}$.

Data are presented as mean \pm SEM. Statistical analysis: Student's $t$ test, ${ }^{*} \mathrm{p}<0.05$, $* * \mathrm{p}<0.01, * * * \mathrm{p}<0.001$ 
Table 1. Body weight of mice before and after treatment with vehicle or MS-275

\begin{tabular}{|c|c|c|c|c|c|}
\hline & BW before treatment & BW after treatment & Weight loss (gr) & $\%$ of weight loss & Average of BW loss (\%) \\
\hline \multirow{15}{*}{ Vehicle } & 39.32 & 38.57 & 0.75 & 1.9 & 7.8 \\
\hline & 35.09 & 30.02 & 5.07 & 14.4 & \\
\hline & 37.21 & 35.85 & 1.36 & 3.7 & \\
\hline & 46.15 & 44.55 & 1.6 & 3.5 & \\
\hline & 39.55 & 36.64 & 2.91 & 7.4 & \\
\hline & 36.12 & 34.39 & 1.73 & 4.8 & \\
\hline & 39.74 & 33.6 & 6.14 & 15.5 & \\
\hline & 40.9 & 39.05 & 1.85 & 4.5 & \\
\hline & 35.67 & 31.86 & 3.81 & 10.7 & \\
\hline & 34.36 & 33.02 & 1.34 & 3.9 & \\
\hline & 45.04 & 42.56 & 2.48 & 5.5 & \\
\hline & 47.94 & 43.02 & 4.92 & 10.3 & \\
\hline & 43.23 & 37.54 & 5.69 & 13.2 & \\
\hline & 41.05 & 37.43 & 3.62 & 8.8 & \\
\hline & 38.27 & 35.04 & 3.23 & 8.4 & \\
\hline \multirow{15}{*}{ MS-275 } & 35.36 & 33.15 & 2.21 & 6.3 & 15.5 \\
\hline & 43.7 & 38.82 & 4.88 & 11.2 & \\
\hline & 41.07 & 29.56 & 11.51 & 28.0 & \\
\hline & 39.16 & 34.45 & 4.71 & 12.0 & \\
\hline & 39.83 & 31.84 & 7.99 & 20.1 & \\
\hline & 40.68 & 35.22 & 5.46 & 13.4 & \\
\hline & 43.75 & 38.41 & 5.34 & 12.2 & \\
\hline & 33.7 & 28.82 & 4.88 & 14.5 & \\
\hline & 41.07 & 37.58 & 3.49 & 8.5 & \\
\hline & 41.37 & 34.22 & 7.15 & 17.3 & \\
\hline & 38.54 & 33.98 & 4.56 & 11.8 & \\
\hline & 52.76 & 46.18 & 6.58 & 12.5 & \\
\hline & 40.35 & 31.92 & 8.43 & 20.9 & \\
\hline & 36.23 & 29.26 & 6.97 & 19.2 & \\
\hline & 43.76 & 33.22 & 10.54 & 24.1 & \\
\hline
\end{tabular}

(C) 2016 Macmillan Publishers Limited. All rights reserved. 


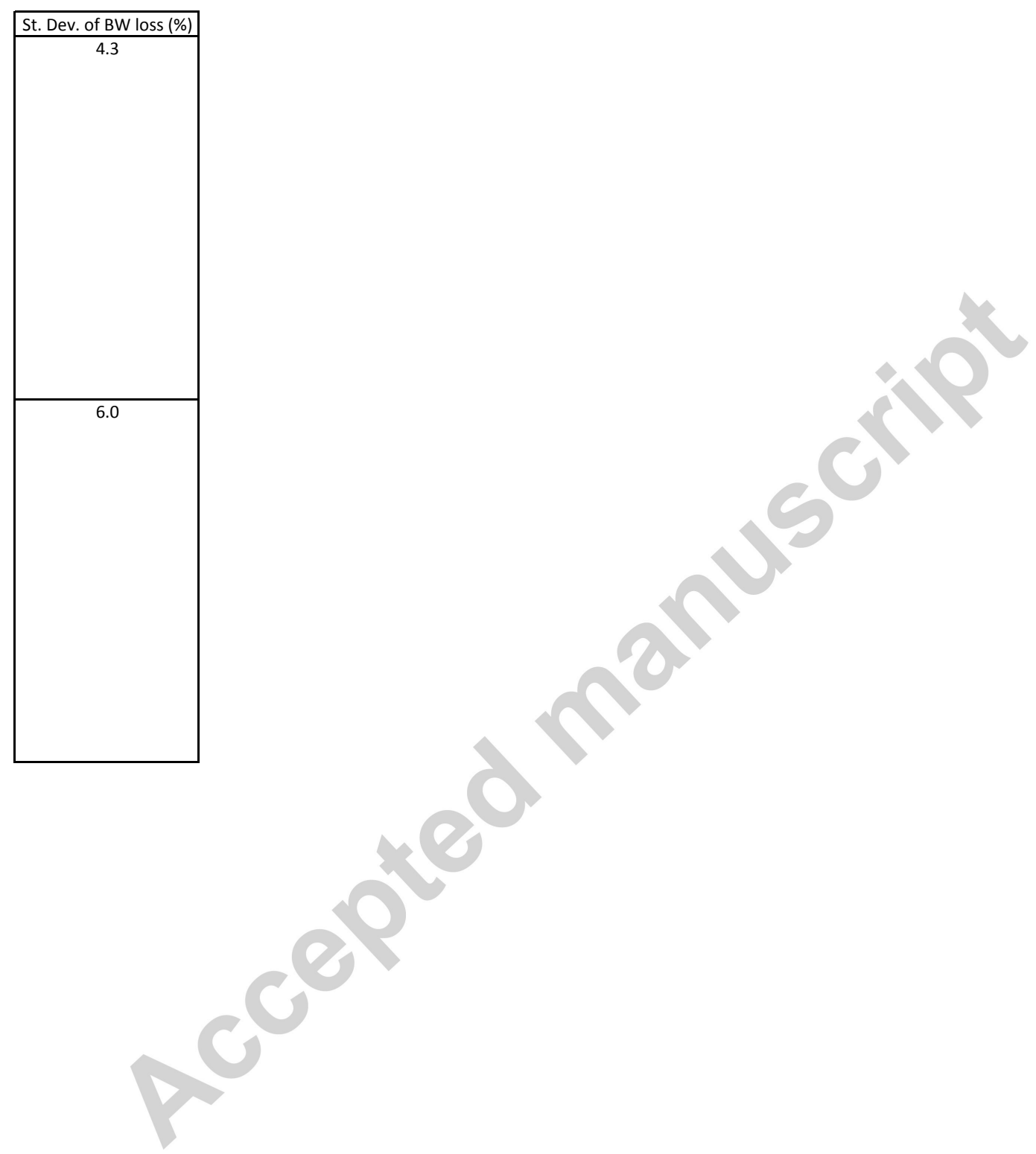

(C) 2016 Macmillan Publishers Limited. All rights reserved. 
Figure 1

a

HFD (60\% calories from fat)

\section{0 weeks}

b

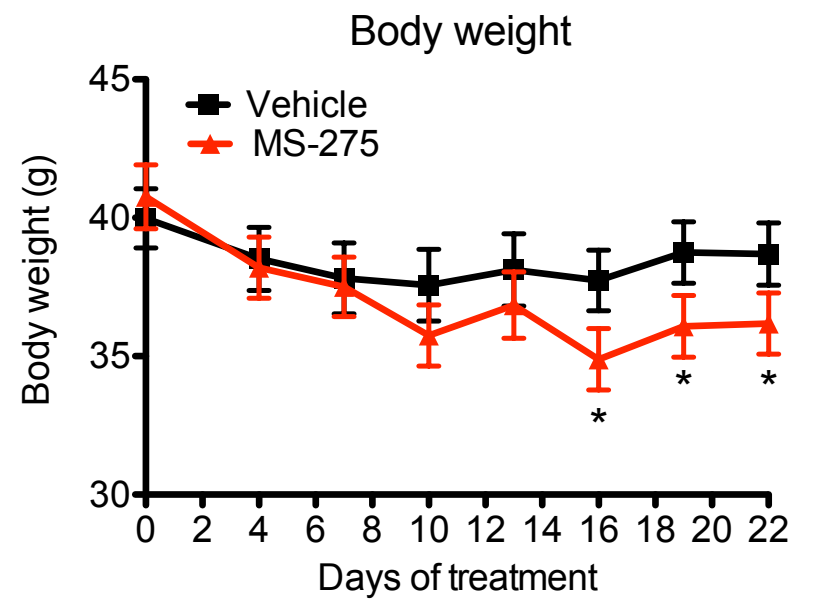

\section{c}

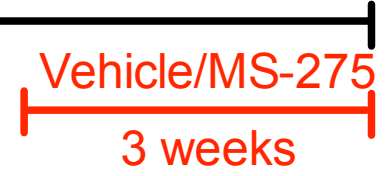

$\%$ WAT

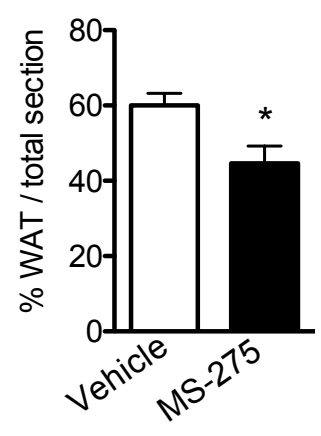

AUC

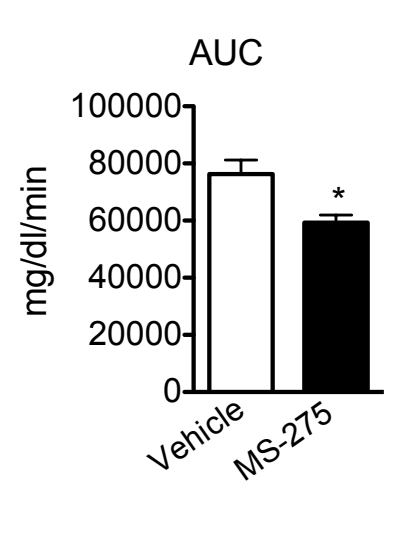

e
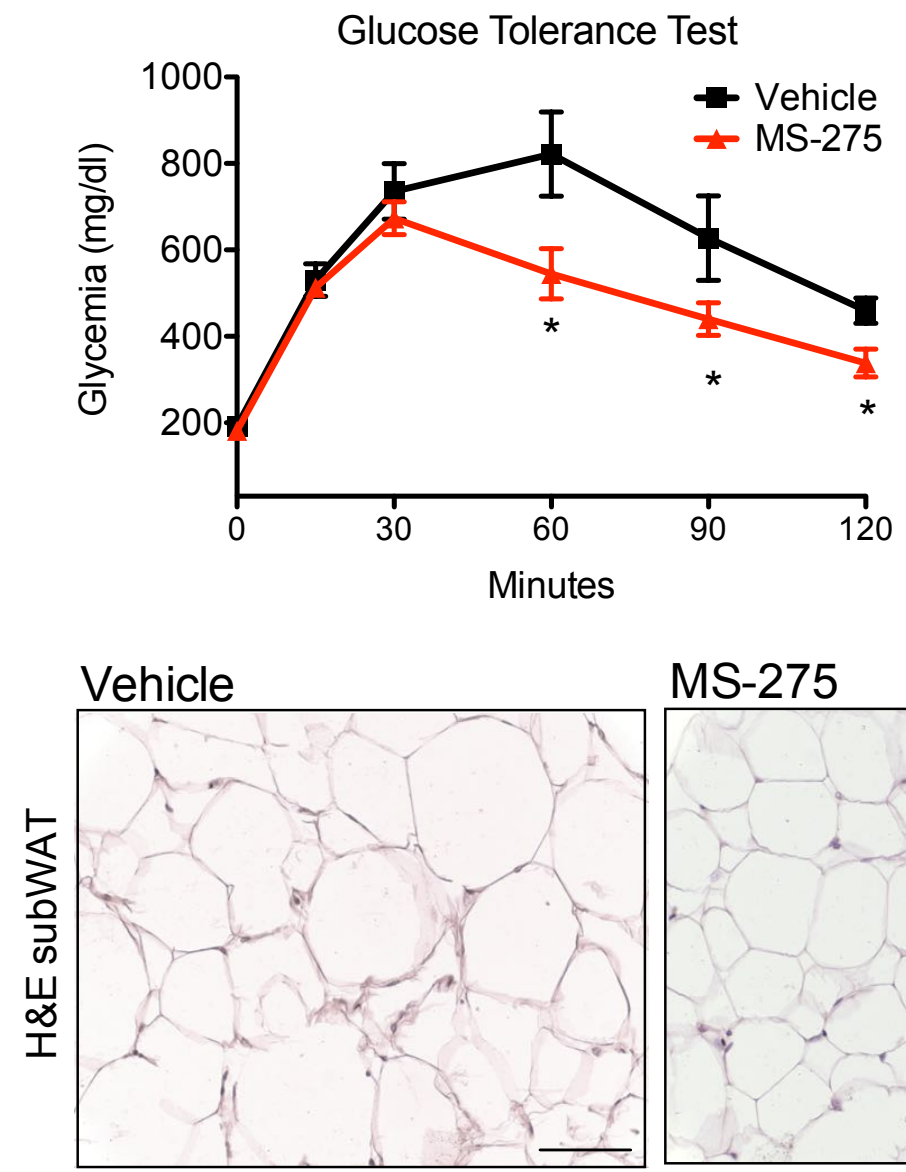

MS-275
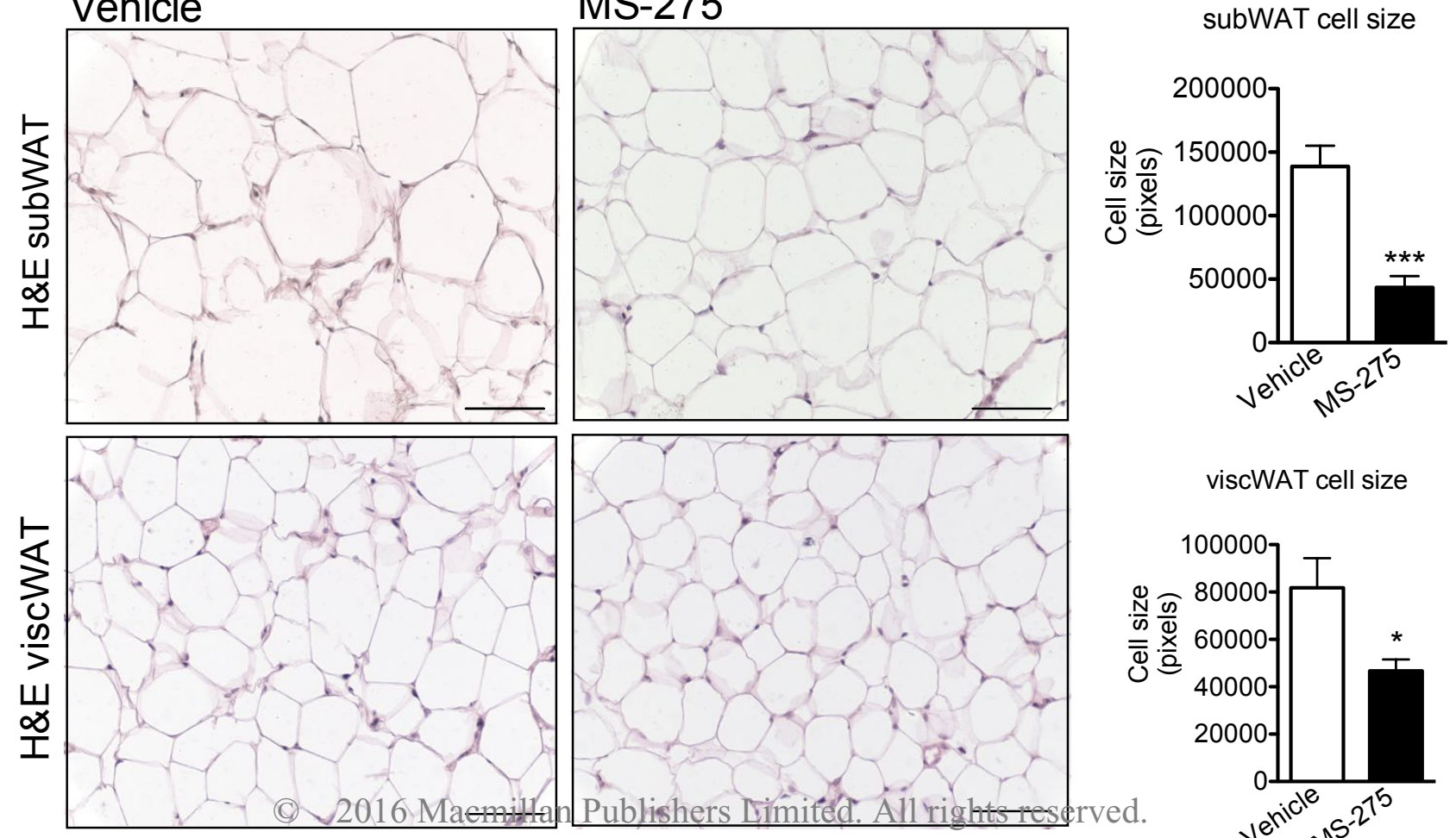

viscWAT cell size

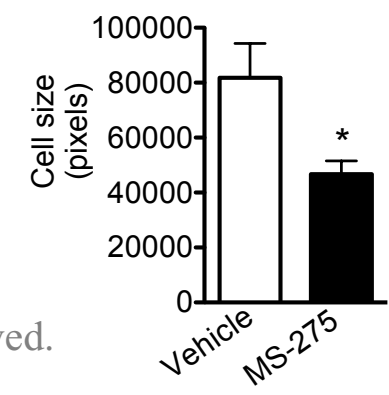




\section{Figure 2}

a

SubWAT: Functionality markers

ViscWAT: Functionality markers
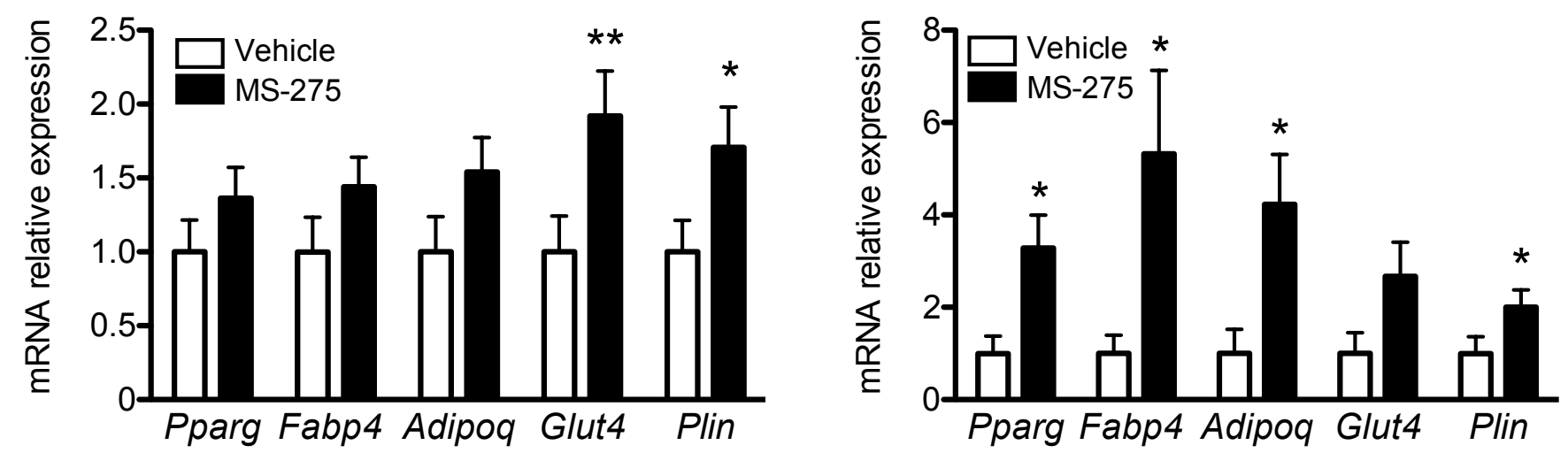

C
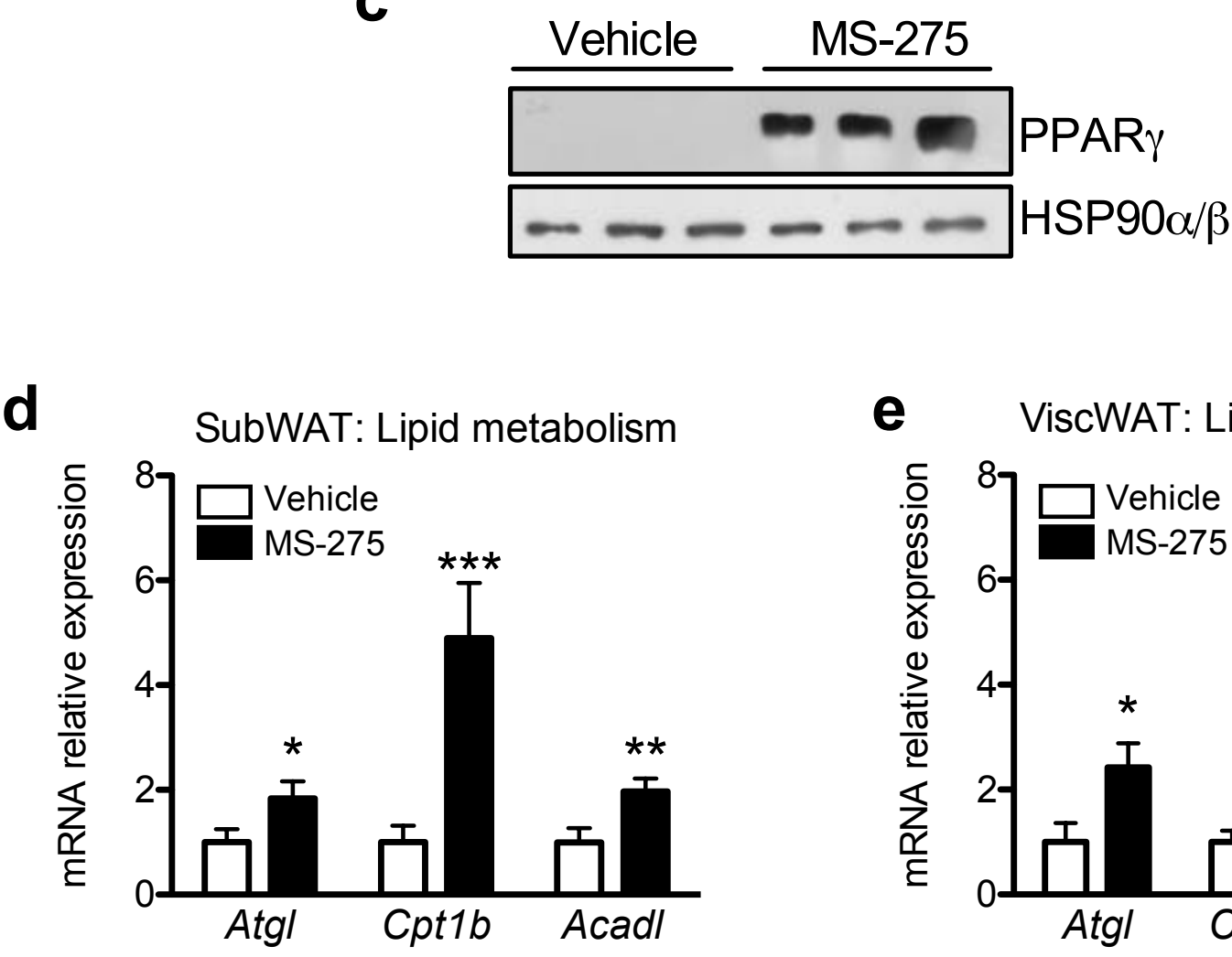

e ViscWAT: Lipid metabolism

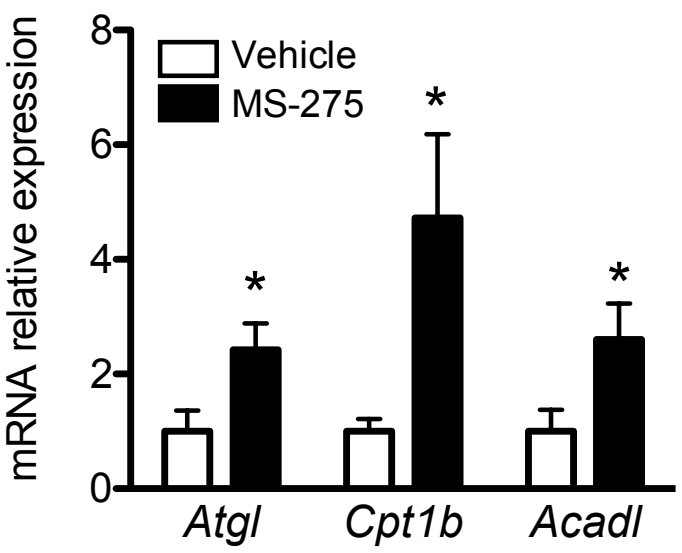

f

NEFA

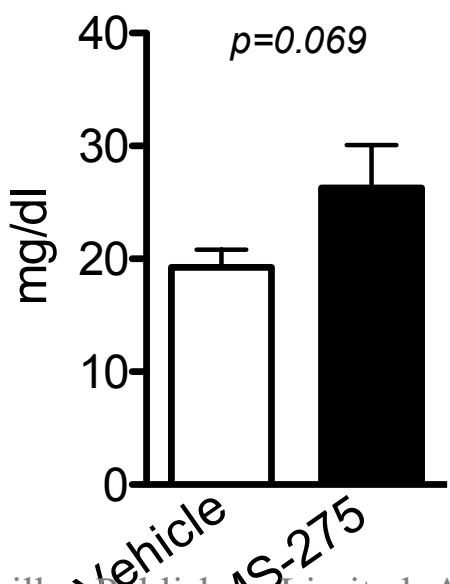




\section{Figure 3}

a
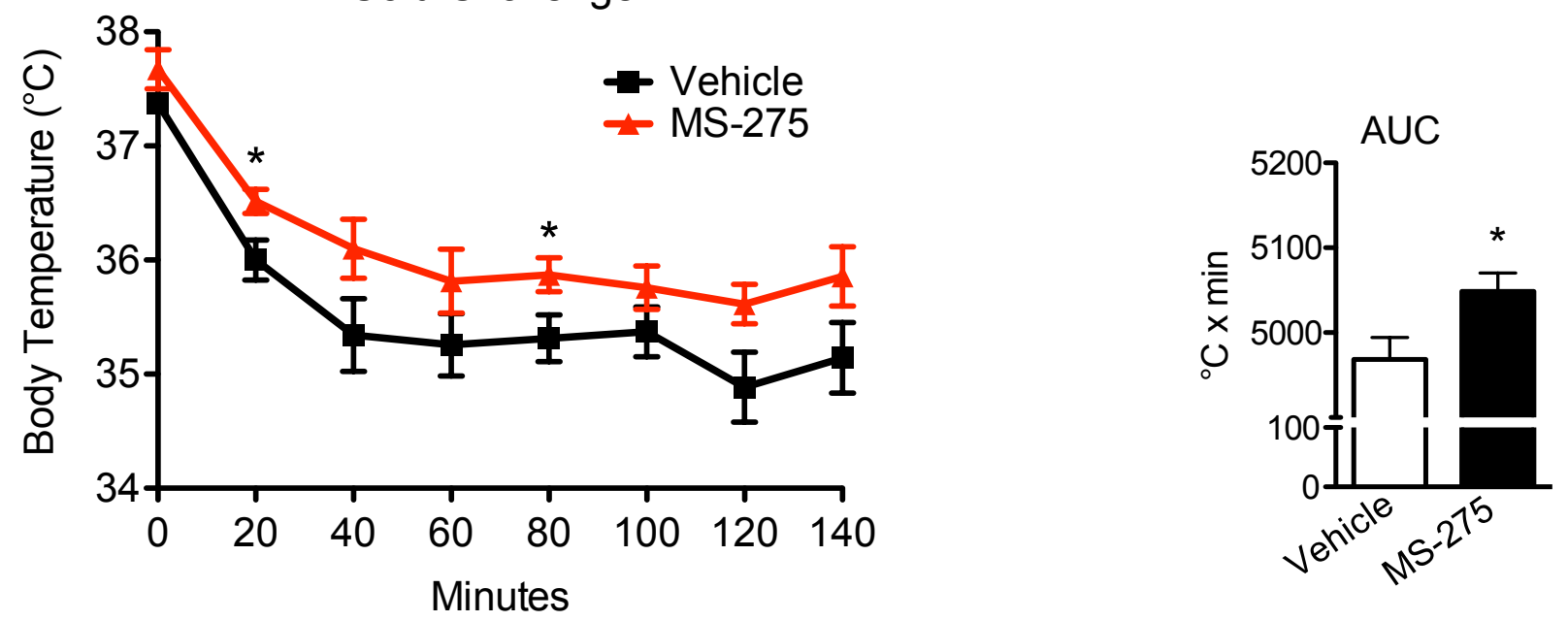

b

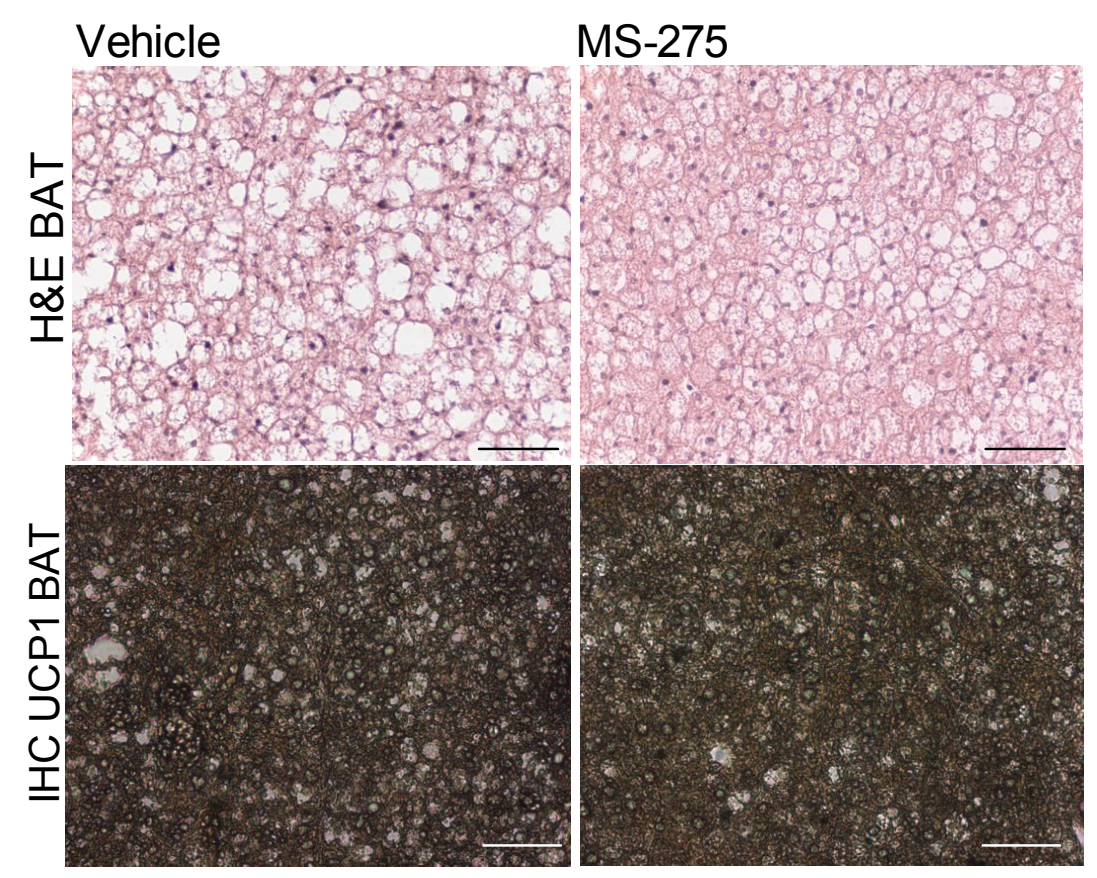

C

Brown fat specific genes
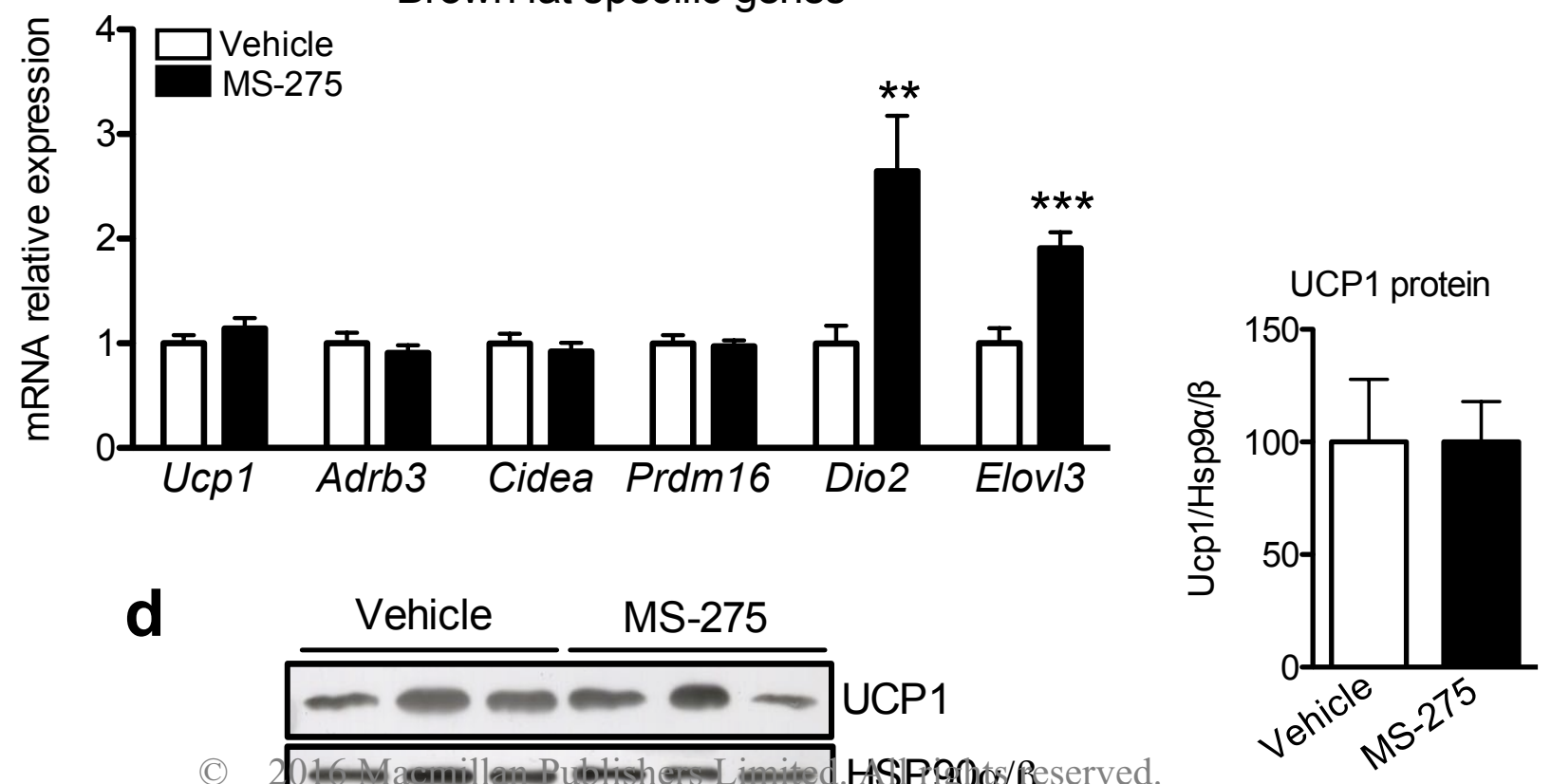


\section{Figure 4}
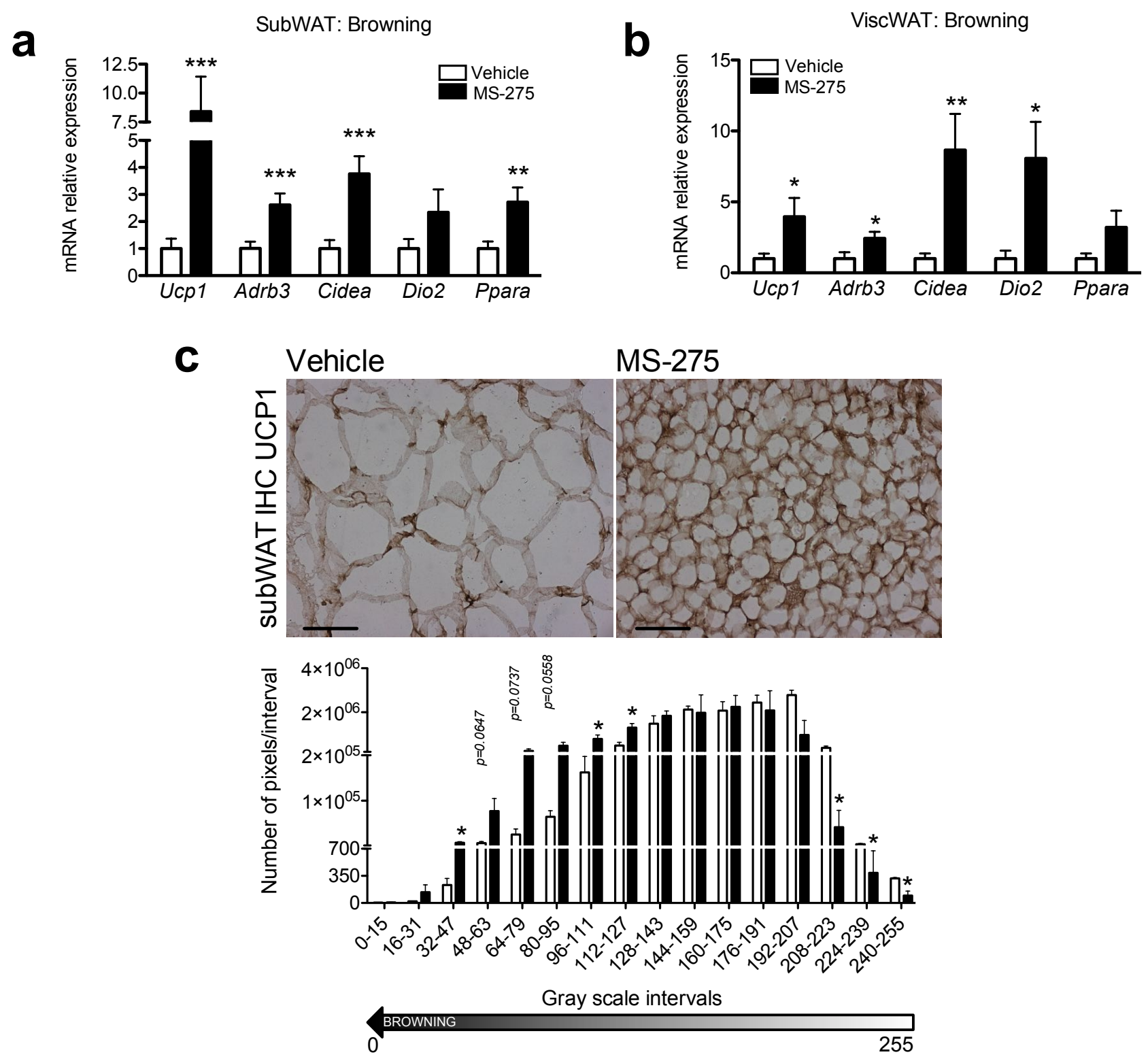

d
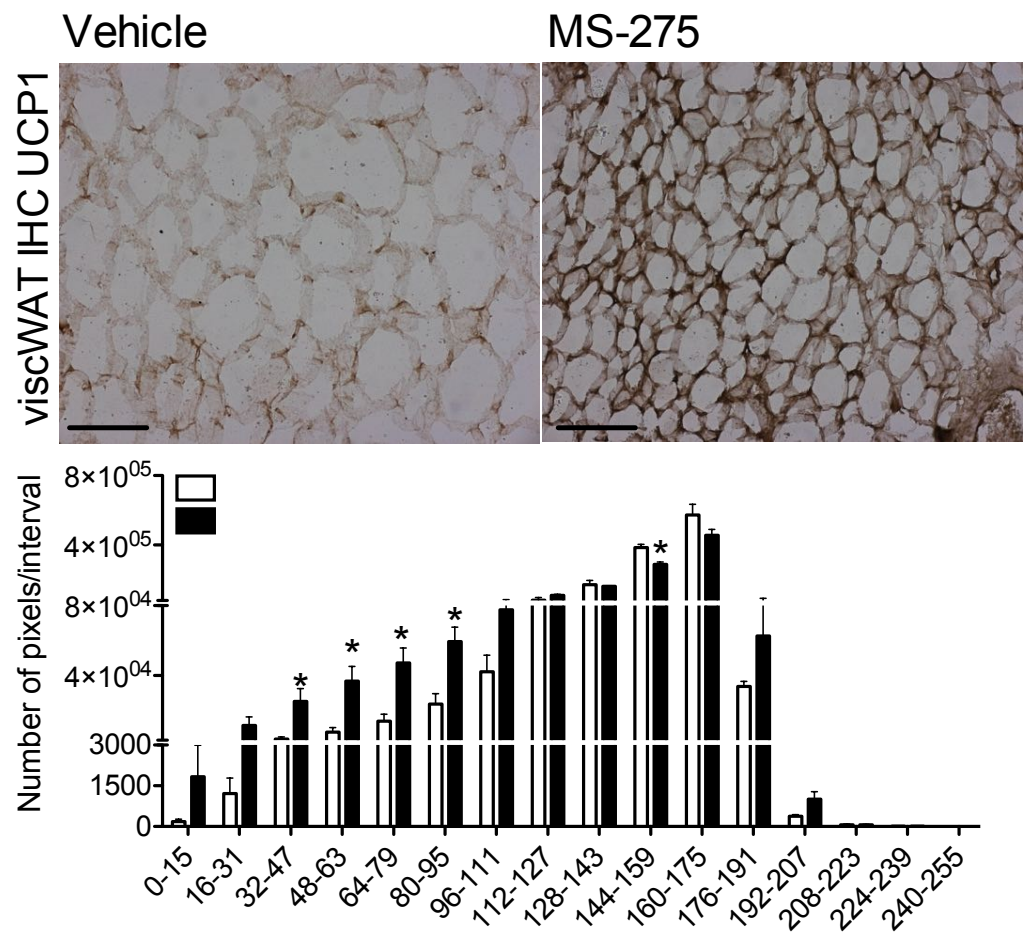

(C) $2016 \underset{0}{\text { Machowillagn_Publishays Gcale intervals }}$ 